\title{
Sex-related inequalities in management of patients with acute coronary syndrome-results from the EURHOBOP study
}

\author{
Carla Araújo $^{1,2}$ (D) | Marta Pereira ${ }^{1}$ | Olga Laszczyńska ${ }^{1}$ | Paula Dias $^{3}$ | Ana Azevedo ${ }^{1,4}$
}

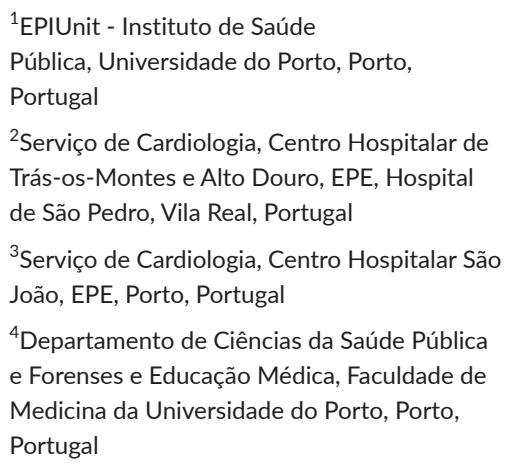

\begin{abstract}
Summary
Background: Real-world data from different levels of hospital specialisation would help to understand if differences in management between women and men with acute coronary syndrome (ACS) are still a priority target. We aimed to identify sex inequalities in management of patients with different types of ACS.

Methods: We analysed 1757 patients with a non-ST-elevation ACS (NSTEACS) and 1184 with ST elevation myocardial infarction (STEMI) or left bundle branch block (non-classifiable (NC) ACS (STEMI/NC ACS group), consecutively discharged from ten Portuguese hospitals with different specialisation levels, between 2008 and 2010. We estimated odds ratios (OR) and $95 \%$ confidence intervals $(95 \% \mathrm{Cl})$ for the association between sex and the performance of coronary angiography, reperfusion and revascularisation.

Results: Among STEMI/NC ACS, men had higher probability of performing coronary angiography than women (adjusted $\mathrm{OR}=1.64,95 \% \mathrm{Cl}$ : 1.11-2.44), while among NSTEACS patients there was no significant difference by sex (adjusted $O R=1.26$, 95\% Cl: 0.99-1.62). In patients who underwent coronary angiography, there was no difference in proportion of women and men submitted to revascularisation, regardless of the ACS type. Although men with STEMI/NC ACS were more likely to undergo reperfusion (crude $\mathrm{OR}=2.17,95 \% \mathrm{Cl}: 1.68-2.81$ ), the effect became not significant after multivariable adjustment (adjusted $\mathrm{OR}=1.33,95 \% \mathrm{Cl}$ : 0.96-1.84).

Conclusion: Women diagnosed with STEMI/NC, but not NSTEACS, had lower probability when compared with men to be submitted to coronary angiography. There was no difference in performance of reperfusion and revascularisation by sex.
\end{abstract}

\section{1 | INTRODUCTION}

In the last decades, one of the major determinants of the decrease in coronary heart disease (CHD) mortality in Europe, in women and men, was a more effective access to coronary angiography. ${ }^{1}$ However, recent data show that men with an acute coronary syndrome (ACS) are still more likely to undergo an invasive approach. ${ }^{2}$ In Portugal, between 2010 and 2013, 16.5\% of patients with non-ST-elevation myocardial infarction, mostly women, were treated with a conservative strategy. ${ }^{3}$ Although from 2002 to 2013 a three-fold increase in primary angioplasty was observed, the proportion of women remained approximately the same $\left(24.5 \%\right.$ and $26.2 \%$, respectively). ${ }^{4}$ These data were obtained from registries that included patients admitted to cardiology departments, but not to other departments, and to hospitals offering differentiated care, and therefore might not represent the national trends in the invasive diagnostic-therapeutic approach of women and men with ACS.

The decrease in CHD mortality in Portugal was found to be greater among women compared to men, ${ }^{5}$ the result of effective primary and/ or secondary prevention measures implemented in this group. Changes in the major cardiovascular risk factors in Portugal contributed significantly more to the CHD decrease among women than among men, mainly due to lifestyle changes, as the effect of risk factor lowering treatment was small and similar between sexes. ${ }^{1}$ Improvements in sex 
differences in discharge medications after an ACS as well as in drugeluting stents use might also have contributed to these results. ${ }^{6,7}$

Sex and gender differences concerning ACS are described in several categories, namely risk assessment, disease awareness, comorbidities, presentation, treatment and outcomes, ${ }^{8}$ contributing to a potential different access to health care between women and men. Real-world data about sex differences in management of ACS patients, treated in hospitals with diverse characteristics, and considering possible relative contraindications to an invasive approach and to revascularisation, represent an operational measurement of effective access. We aimed to analyse sex differences in management of ACS, controlling for age, hospital characteristics, cardiovascular risk factors, previous medical history, complications at admission and coronary anatomy, within a sample of Portuguese hospitals serving both urban and rural populations and with different levels of specialisation.

\section{2 | METHODS}

\section{1 | Study design and sample selection}

Data for this study were collected within the framework of the EURopean Hospital Benchmarking by Outcomes in acute coronary syndrome Processes (EURHOBOP) project, a collaborative, multicentre and multinational retrospective study of patients consecutively hospitalised with a discharge diagnosis of ACS from 70 hospitals in 7 European countries (Finland, France, Germany, Greece, Italy, Portugal, and Spain), 9,10 For the current analysis we used data from patients admitted to the 10 Portuguese hospitals, which were selected to cover different regions from the mainland country, from north to south and west to east and including both coastal and interior regions, urban and rural populations. Furthermore, these hospitals had different characteristics, regarding population served, facilities, technical and human resources, and specialisation levels. These diverse settings were selected with the purpose of seeking representativeness of the general ACS population. Overall five hospitals had a catheterisation laboratory, three had a cardiac surgery department, in one patients with ACS were admitted to the internal medicine department, as the hospital had no cardiology department or cardiologists, four were university hospitals, the number of beds ranged from 280 to 1124 and the populations served ranged from less than 300000 to more than 700000 people. Each hospital contributed with approximately 300 consecutive patients discharged between 2008 and 2010 with diagnosis of ACS (International Classification of Diseases 10th revision: I.21.0-I 21.9 and I.20.0). From 3009 ACS patients included, those with missing data on the type of ACS were excluded $(n=68)$.

\section{2 | Procedures and data collection}

With the use of standardised forms, trained investigators extracted data from discharge letters, emergency room records and laboratory systems. When necessary, different sources were cross-checked to ensure completeness and quality of the information. Information on type of ACS, demographic characteristics, previous medical history, admission data, procedures used during hospitalisation, severity

\section{What is known}

- Several studies have suggested that women with acute coronary syndrome are more likely to be treated with a conservative strategy than men.

- Studies in different settings support that disparities in care and outcomes of women and men persisted over time.

- Whether this sex-gap in management is observed for diagnostic or also for invasive therapeutic coronary procedures; and for the whole spectrum of presentations of acute coronary syndrome, managed in hospitals with different levels of specialisation is controversial.

\section{What is new}

- Women with ST-elevation acute myocardial infarction/ non-classifiable (left bundle branch block) acute coronary syndrome, but not with non-ST-elevation acute coronary syndrome, were less frequently submitted to coronary angiography than men.

- No differences in reperfusion or revascularisation among those managed invasively were observed between women and men for the whole spectrum of acute coronary syndrome presentations.

indicators and complications during hospitalisation, including vital status and in-hospital medication (the main classes of recommended drugs for patients with $\mathrm{ACS})^{11,12}$ was extracted.

\section{3 | Definition of variables and data analysis}

Patients with left bundle branch block were defined as non-classifiable (NC) ACS (NC ACS) and analysed with STEMI patients (STEMI/NC ACS group)

The management was considered invasive if coronary angiography was performed. Reperfusion was defined as either thrombolysis or primary percutaneous coronary intervention; revascularisation, either as percutaneous coronary intervention $(\mathrm{PCl})$ or coronary artery bypass graft (CABG). Anaemia was defined as haemoglobin below $12 \mathrm{~g} / \mathrm{dL}$ for females and below $13 \mathrm{~g} / \mathrm{dL}$ for males ${ }^{13}$ and renal impairment was subdivided in two groups: estimated glomerular filtration rate (eGFR) below $30 \mathrm{~mL} / \mathrm{min} / 1.73 \mathrm{~m}^{2}$ and between 30 and $60 \mathrm{~mL} /$ $\mathrm{min} / 1.73 \mathrm{~m}^{2} \cdot{ }^{14}$ Angiographic coronary disease was defined as normal/ mild if coronary arteries were normal or with stenosis $<30 \%$; moderate for stenosis between $30 \%$ and $70 \%$; and severe for any obstruction $>70 \%$ or $>50 \%$ if the obstruction was in the left main coronary artery. Severe coronary disease was further divided into 1-, 2- and 3-vessels disease categories, according to the number of affected arteries. Inhospital complications were evaluated by sex and type of ACS through a composite endpoint of pulmonary oedema, shock, acute renal failure, re-infarction, stroke and a drop of haemoglobin of 3 or more $\mathrm{g} / \mathrm{dL}$. In-hospital death was also assessed separately by sex and type of ACS. 
The proportions of prescription of main classes of drugs during hospitalisation were assessed for eligible patients, according to sex, type of ACS and management approach, namely invasive vs conservative (according to the performance or not of coronary angiography). We computed composite variables for drugs combinations: double antiplatelet therapy with aspirin and clopidogrel, 3-drug treatment [(aspirin or clopidogrel) and beta-blocker and statin] and 5-drug treatment [aspirin and clopidogrel and beta-blocker and (angiotensin-converting enzyme inhibitors (ACEi) or angiotensin receptor blockers (ARB) and statin]. For simple antiplatelet therapy (aspirin or clopidogrel), haemoglobin below $10 \mathrm{~g} / \mathrm{dL}$ for females and below $11 \mathrm{~g} / \mathrm{dL}$ for males at admission was considered a contraindication. For double antiplatelet therapy, besides a low value of haemoglobin at admission, previous atrial fibrillation or being on oral anticoagulation at admission were considered also contraindications. A systolic blood pressure lower than $100 \mathrm{~mm} \mathrm{Hg}$ or severe renal failure (eGFR less than $30 \mathrm{ml} /$ $\min / 1.73 \mathrm{~m}^{2}$ ) at admission were considered contraindications for ACEi or ARB. Contraindications for beta-blockers were systolic blood pressure lower than $100 \mathrm{~mm} \mathrm{Hg}$ or heart rate below 50 bpm at admission.

Potential confounders of the association between sex and management were grouped as follows: hospital characteristics, cardiovascular risk factors, cardiovascular history, complications at admission and angiographic coronary disease (the latter for the revascularisation models only). In the univariate analysis, we identified which variables were associated with management $(P$-value $<.15)$, using logistic regression. Within each group mentioned above, variables with a significant effect on the dependent variable were included in the multivariate model and a backward strategy was used to exclude the least significant variables, based on Wald test ( $P$-value $<.05)$. To fit the final model, we departed from all variables with significant effect on outcome derived from the intragroup multivariate modelling, repeated backward strategy to choose the significant variables and performed likelihood ratio test for boundary $\mathrm{p}$ value. In sensitivity analysis, we examined the sex differences in management for STEMI group excluding the NC ACS patients. Data were analysed, using STATA version 11 for Windows (Stata Corp LP, College Station, TX, USA).

\section{4 | Ethics}

The study was approved by the ethics committee of the University of Porto Medical School and the National Commission for Data Protection. These two entities agreed that it would not be necessary to ask for patients' informed consent, since the study was based on the collection of retrospective clinical data from the medical records during hospitalisation, and the confidentiality of patients' identification was assured.

\section{3 | RESULTS}

\section{1 | Baseline characteristics}

A total of 2941 patients were included, 1757 (59.7\%) with NSTEACS diagnosis and 1184 (40.3\%) with STEMI/NC ACS (Tables 1 and 2).
The proportions of women included by hospital varied between $27.5 \%$ and $39.2 \%$ and the mean age of the patients between 64.1 and 70.2 years old (Table 1 ). In the majority of the hospitals, the proportion of patients with STEMI/NC ACS was above 40\%, however, in three hospitals lower proportions were observed; the lowest was $24.7 \%$ in a hospital without catheterisation laboratory and cardiology ward (Table 1). Only in one hospital, the proportion of patients with previous history of myocardial infarction, $\mathrm{PCl}$ or CABG was below $20 \%$. The proportions of patients with complications at admission varied between $38.7 \%$ and $61.1 \%$ between hospitals. The two hospitals with the lowest and the highest proportions of complications at admission were similar in characteristics, namely were tertiary hospitals, with catheterisation laboratory, located in the coast and covering an urban predominantly population. In all hospitals, most patients had severe angiographic coronary disease of at least of one vessel. The proportion of patients who had at least one in-hospital complication varied between $7.3 \%$ in Hospital de Faro and $23.7 \%$ in Centro Hospitalar do Porto. The in-hospital mortality varied between $0.8 \%$ in Centro Hospitalar Alto Ave and $14.6 \%$ in Centro Hospitalar Cova da Beira, the latter is located in the interior, had no catheterisation laboratory and cardiology ward, therefore patients with ACS were admitted to the internal medicine department (Table 1).

Compared to men, women with either ACS type were older, had more frequently hypertension and diabetes and were less frequently current smokers. Women had more comorbidities than men, independently of the ACS type: they suffered more often from previous heart failure, atrial fibrillation and renal failure. In the NSTEACS population, previous myocardial infarction, $\mathrm{PCl}$ or $\mathrm{CABG}$ were more prevalent in men. Anaemia and renal impairment at admission were more prevalent in women, who presented less often with severe angiographic coronary disease (Table 2). Compared with men, the composite endpoint of in-hospital complications (pulmonary oedema, shock, acute renal failure, reinfarction, stroke, drop in haemoglobin of $3 \mathrm{~g} / \mathrm{dL}$ or more) was more frequently observed among women with NSTEACS (15.3\% vs $11.2 \%, P=.014)$ and with STEMI/NC ACS (19.7\% vs $13.8 \%$, $P=.011)$. Of all patients included in this analysis, $5.1 \%$ of women and $3.7 \%$ of men with NSTEACS died during hospitalisation $(P=.159)$, while among STEMI/NC ACS patients, in-hospital death was significantly higher among women than men $(17.9 \%$ vs $8.3 \%, P<.001$, respectively) (Table 2).

\section{2 | Management}

Compared with patients treated conservatively, women and men with NSTEACS or STEMI/NC ACS who were treated invasively more frequently had prescription of the several recommended classes of drugs during hospitalisation. Patients with STEMI/NC ACS, both women and men, managed conservatively were the subgroup who had the lowest prescription of recommended drugs during hospitalisation (Figure 1). Women with NSTEACS managed conservatively were significantly less likely to receive statins $(54.2 \%$ vs $64.7 \%$, $P=.009)$ and 5 -drug treatment $(20.7 \%$ vs $34.3 \%, P=.002)$ than 


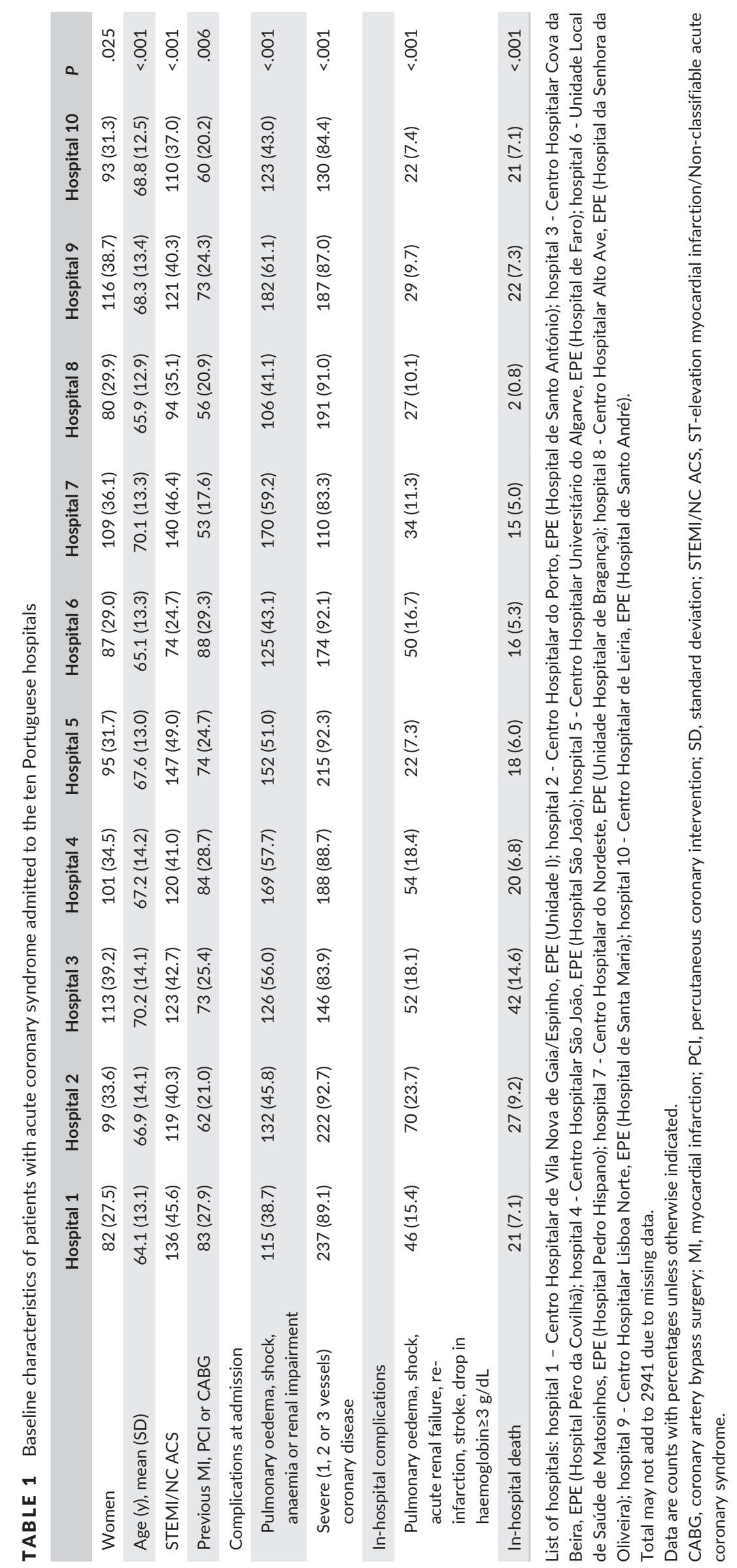


TABLE 2 Baseline characteristics of patients with different types of acute coronary syndrome, by sex

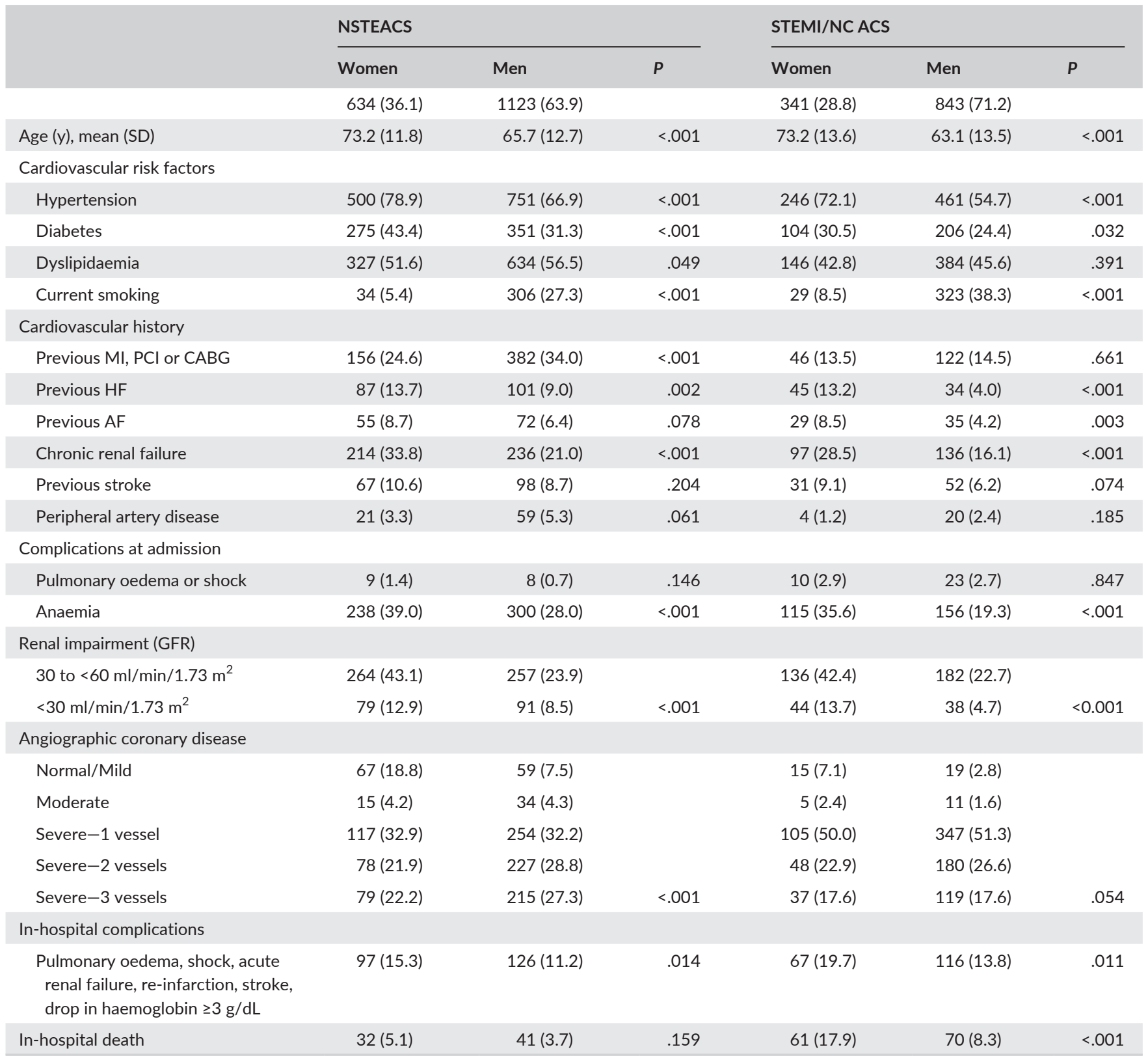

Total may not add to 2941 due to missing data.

Data are counts with percentages unless otherwise indicated.

AF, atrial fibrillation; CABG, coronary artery bypass surgery; GFR, glomerular filtration rate; HF, heart failure; MI, myocardial infarction; NSTEACS, non-STelevation acute coronary syndrome; PCI, percutaneous coronary intervention; SD, standard deviation; STEMI/NC ACS, ST-elevation myocardial infarction/ Non-classifiable acute coronary syndrome.

men with NSTEACS managed conservatively; while women with NSTEACS managed invasively were more likely to receive an ACEi/ ARB during hospitalisation than men with the same diagnosis and management approach (69.3\% vs $61.9 \%, P=.037$, respectively). The remaining sex differences in prescription of drugs during hospitalisation among patients without contraindications were not significant (Figure 1).

An invasive strategy was less frequent in women, regardless of the type of ACS $(56.6 \%$ vs $71.8 \%, P<.001$, and $62.5 \%$ vs $80.8 \%$,
$P<.001$ among patients with NSTEACS and STEMI/NC ACS, respectively). The difference in the odds of being managed invasively between sexes was observed in the STEMI/NC ACS group, after adjustment for patient and hospital characteristics (adjusted odds ratio [OR] 1.64, 95\% confidence interval [95\% Cl] 1.11-2.44). Among patients performing coronary angiography, $52.7 \%$ and $76.5 \%$ of women and $58.3 \%$ and $81.6 \%$ of men with NSTEACS and STEMI/ NC ACS, respectively, were submitted to revascularisation. There was no difference by sex in the odds of revascularisation in both 


\section{NSTEACS}
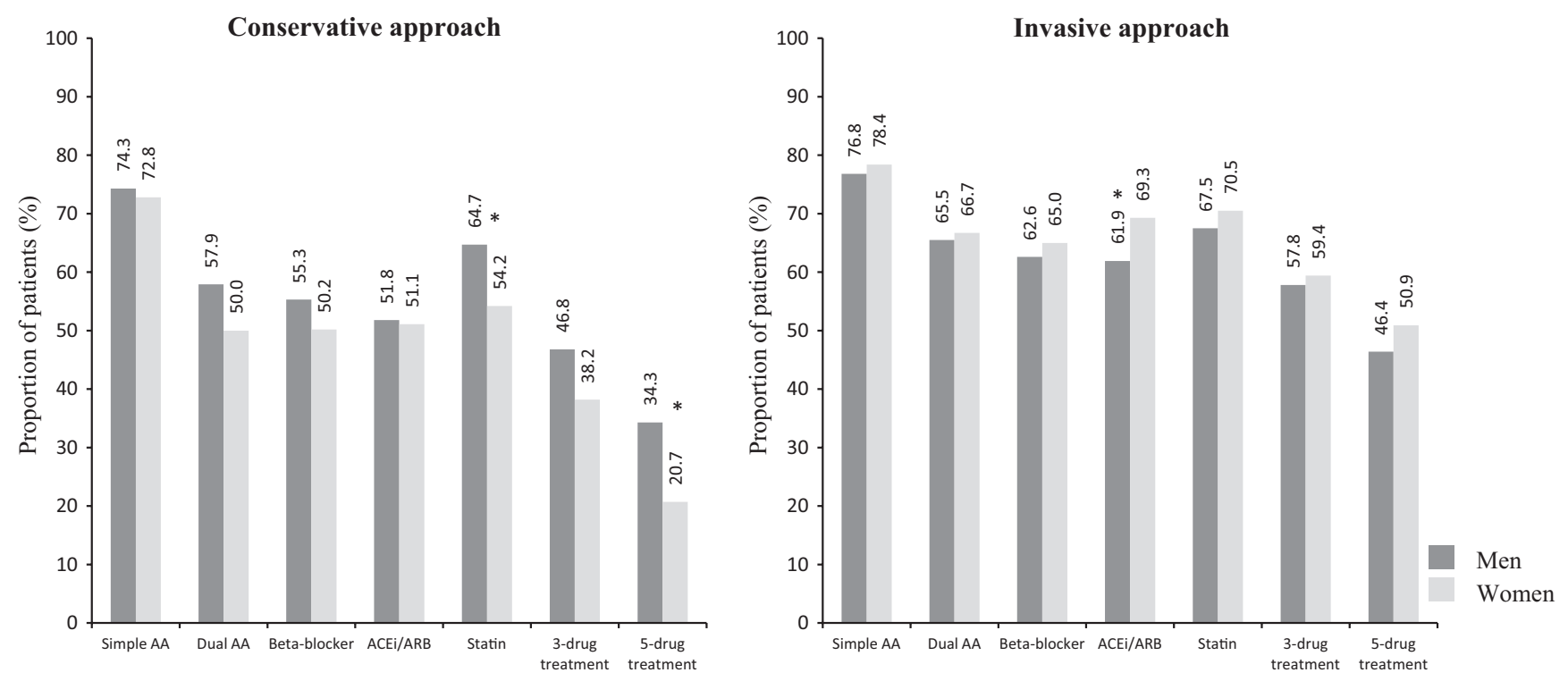

STEMI/NC ACS
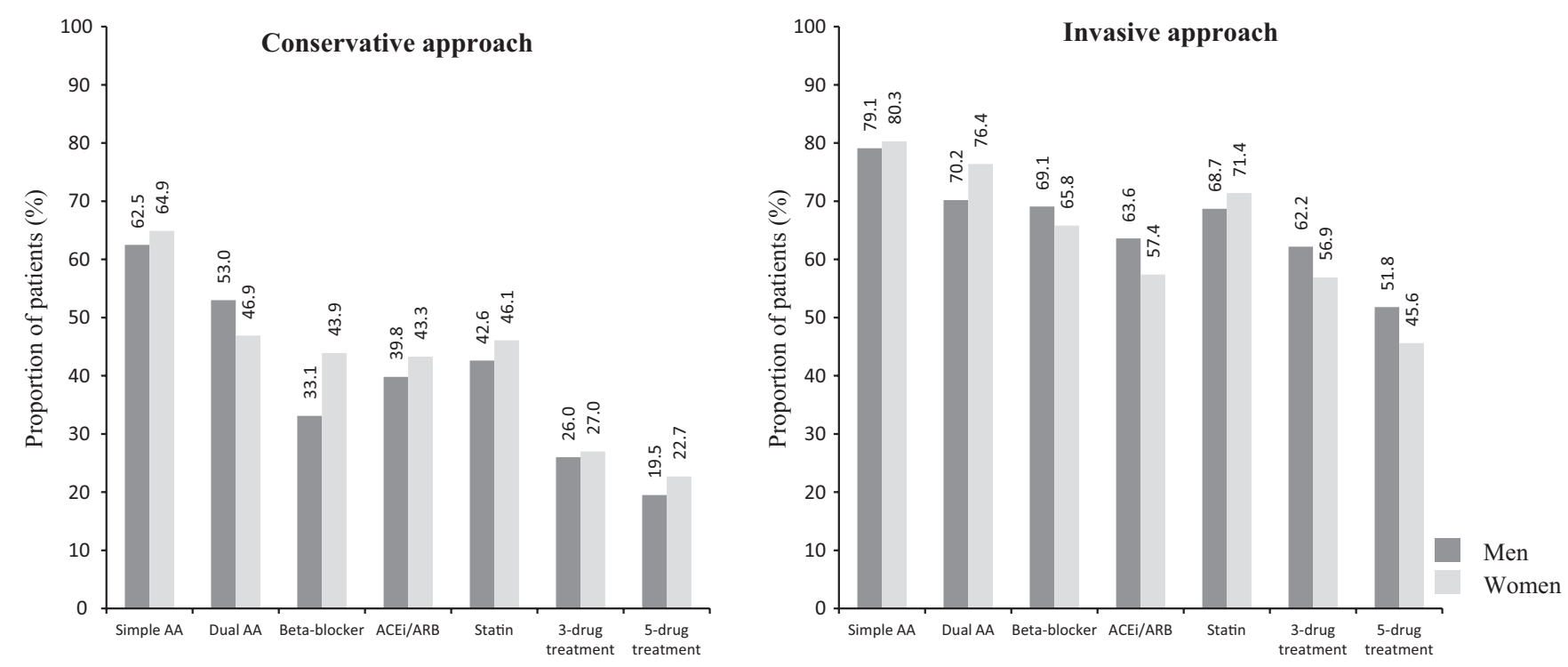

$*$-value $<.05$

ACEi, angiotensin converting enzyme inhibitor; ARB, angiotensin receptor blocker; NSTEACS, non-ST elevation acute coronary syndrome;

STEMI/NC ACS, ST-elevation myocardial infarction/non-classifiable acute coronary syndrome

3-drug treatment (aspirin or clopidogrel) and beta-blocker and statin

5-drug treatment aspirin and clopidogrel and beta-blocker and (ACEi or ARB) and statin

FIGURE 1 Proportion of patients treated with pharmacological treatment during hospitalisation for acute coronary syndrome with or without ST-elevation according to sex and management approach.

NSTEACS (0.84, 0.61-1.14) and STEMI/NC ACS (1.00, 0.62-1.62) after adjustment (Table 3). More men than women with STEMI/ NC ACS had reperfusion therapy (67.9\% vs $49.3 \%$, respectively). However, this significant crude association between male sex and reperfusion $(2.17,1.68-2.84)$ was explained after multivariable adjustment $(1.33,0.96-1.84)$. In the sensitivity analysis, NC ACS patients $(n=163$ ) were excluded and the results were comparable to primary results (Table 4 ).

\section{DISCUSSION}

This study shows that women with STEMI/NC ACS, but not with NSTEACS, were less frequently submitted to coronary angiography than men, after controlling for age, characteristics of the hospitals, cardiovascular risk factors, previous medical history and complications at admission. No significant differences between sexes in the performance of reperfusion and revascularisation were observed. 
TABLE 3 Sex differences in in-hospital management, by type of acute coronary syndrome (women are the reference class)

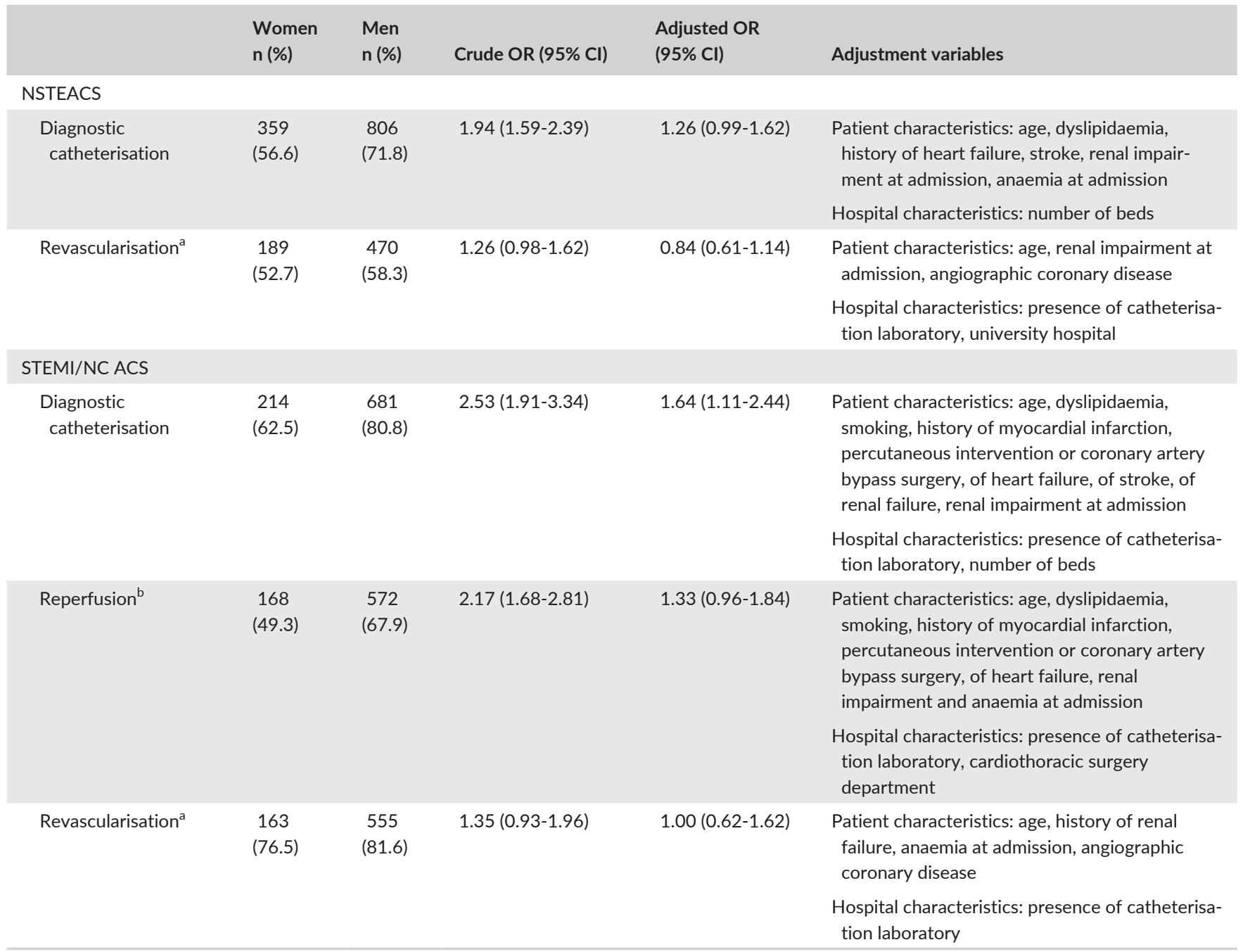

$\mathrm{Cl}$, confidence interval; NSTEACS, non-ST-elevation acute coronary syndrome; OR, odds ratio; STEMI/NC ACS, ST-elevation myocardial infarction/Nonclassifiable acute coronary syndrome.

${ }^{a}$ Percutaneous coronary intervention and/or coronary artery bypass surgery among those submitted to coronary angiography.

${ }^{\mathrm{b}}$ Thrombolysis or primary percutaneous coronary intervention.

We included 10 hospitals with different levels of specialisation, located in heterogeneous geographic areas. The analysis of the characteristics of the patients included by hospital identified differences in ACS epidemiology and outcomes. Between and within country differences in management and outcomes of patients with ACS have been described, but remain poorly understood. ${ }^{10,15,16}$ In Portugal, between 1981 and 2012, relative declines of CHD mortality indicators were different by geographic region; consistent decreases in mortality rates were only observed in the most populated and urbanised regions. ${ }^{5}$ Our finding of significant differences by hospital in in-hospital mortality of patients with ACS deserves further analysis.

Of patients hospitalised with NSTEACS and with STEMI/NCACS $36.1 \%$ and $28.8 \%$, respectively, were women, similar proportions to the observed in the whole EURHOBOP sample. ${ }^{10}$ Approximately $40 \%$ of patients of our sample were diagnosed with STEMI/NC ACS; data from the Portuguese Registry of Acute Coronary Syndrome (ProACS) reported a similar proportion $(41.2 \%) .{ }^{17}$ The prevalence of cardiovascular risk factors in women and men with ACS, higher among patients with NSTEACS is also in line with other national and international data. ${ }^{10,17}$ Particularly relevant is the high prevalence of diabetes in our sample, particularly among women with NSTEACS, higher than the observed in other countries. ${ }^{10}$ For both types of ACS data from the overall EURHOBOP study showed that Portugal had one of the highest proportions of in-hospital events and mortality, even after exhaustive model adjustment. ${ }^{10}$ Analysing data separately by sex and type of ACS, we were able to identify a subgroup of patients with particularly high risk of in-hospital death, women with STEMI/NC ACS (17.9\%). Considering prescription of drugs during hospitalisation, higher differences were observed between patients with different management approaches, than between women and men. The decision to proceed or not to an invasive approach may influence the prescription of recommended drugs for patients with ACS, not only antiplatelet therapy, which is expected, but probably other classes of drugs. Our results are in line with previous findings 
TABLE 4 Sex differences in in-hospital management of patients with ST-elevation myocardial infarction (women are the reference class)

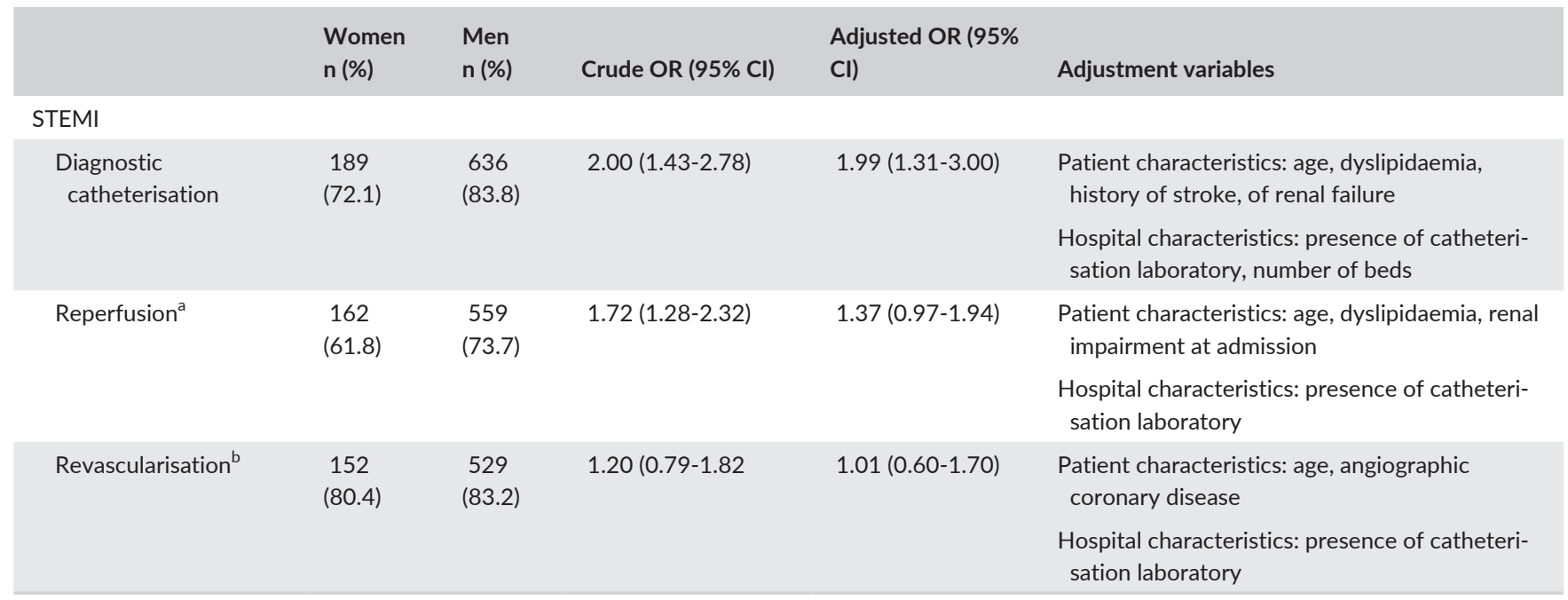

$\mathrm{Cl}$, confidence interval; OR, odds ratio; STEMI, ST-elevation myocardial infarction.

${ }^{\mathrm{a} T h r o m b o l y s i s ~ o r ~ p r i m a r y ~ p e r c u t a n e o u s ~ c o r o n a r y ~ i n t e r v e n t i o n . ~}$

${ }^{b}$ Percutaneous coronary intervention and/or coronary artery bypass surgery among those submitted to coronary angiography.

of very few sex differences in discharge medications of patients with ACS in Portugal, after adjusting for the potential confounding effect of age, comorbidities and contraindications. ${ }^{6}$ Women and men differ in the pathophysiology and mechanisms of coronary heart disease. Women with ACS are on average older than men, suffer from more comorbidities, are more prone to atypical presentations, thrombus formation and plaque erosion, but less prone to suffer from severe obstructive coronary artery disease. ${ }^{18,19}$ Women encounter longer delays between the onset of symptoms and first medical contact, arrival at a hospital and evidence-based treatment. ${ }^{8}$ Additionally although lower cardiac catheterisation rates seen in women with ACS were considered to be partially due to women's own preferences, ${ }^{20}$ recent data suggest that differences in catheterisation rates by sex may be driven largely by physicians, through different patterns of counselling and referral to cardiovascular testing between women and men, and not by differential attitudes, behaviours and decisions of female patients. ${ }^{21}$

Considering access to healthcare in a multilevel perspective, both factors related to health care systems and to patient ${ }^{22}$ explain the difference in management of women and men with ACS. Several determinants, at both levels, that are not independent and influence each other and operate at different times during the process of illness and care, probably enable or hinder differently in women and men, the ability to perceive, seek, reach, pay and engage in health care and the ability of the system to fulfil the needs of the patient. ${ }^{22}$

Compared with data reported by the ProACS from 2010 to 2013, a lower proportion of NSTEACS patients in the EURHOBOP cohort were treated invasively $(66.3 \%$ vs $84.8 \%){ }^{3}$ Although part of this difference may be dependent on a true increase in the invasive treatment of patients with ACS, the results observed in the ProACS may be an overestimation of the true proportion, due to the fact that only hospitals with higher specialisation levels were included in this registry. In NSTEACS patients, only those with unstable angina, without risk criteria and no recurrent symptoms are recommended a non-invasive testing for ischaemia (preferably with imaging) before deciding on an invasive evaluation. ${ }^{11}$ All the others should be treated invasively, unless some contraindication exists or the risks appear to outweigh the benefits. The factors that should be taken into account to weigh risks and benefits are related with clinical presentation, comorbidities, risk stratification, frailty, cognitive status and estimated life expectancy. ${ }^{11}$ After adjusting for several of these factors, no difference in performance of coronary angiography between sexes was observed in the NSTEACS group, representing an improvement in the sex equality of access to evidence-based treatment. ${ }^{2}$

In STEMI patients, the main limitation to reperfusion therapy is the time of presentation after symptoms onset. Early mechanical or pharmacological reperfusion should be performed within the first 12 hours from symptoms onset; whether $\mathrm{PCl}$ is also beneficial in patients presenting more than 12 hours after symptoms onset is more controversial. ${ }^{12}$ The use of reperfusion therapy in our sample is similar to the observed in ProACS covering years $2002-2008$ (62.5\% vs $61.9 \%$, respectively), ${ }^{23}$ but lower than the observed in the second phase of the ProACS (84.8\%). ${ }^{17}$ Implementation of a pre-hospital fast-track network in Portugal improved reperfusion rates in STEMI patients, through an increase in primary angioplasty. ${ }^{4}$ Studies performed with data from the ProACS were not reported by sex, which limits further comparisons. Among STEMI/NC ACS patients, different probabilities of performing coronary angiography by sex might result from distinct patient and hospital delays. ${ }^{8}$ In 2008, Portugal was among the countries performing less primary $\mathrm{PCl}$ in Europe. ${ }^{24}$ One major factor contributing for this finding might be the high proportion (55\%) of patients who were admitted more than 12 hours after symptom onset; ${ }^{4}$ with higher delays being expected among women. The subjective experience of symptoms influences patients' attitudes in help seeking and professionals' interpretation of clinical presentations, thus affecting access to effective health care. ${ }^{25}$ 
In both types of ACS, no differences in revascularisation among those managed invasively were observed between women and men. This also represents a sex-gap improvement in access to care. When the decision to proceed to an invasive approach is made, risks and benefits of revascularisation have already been evaluated. ${ }^{26}$ In our cohort, a significant proportion of patients of both sexes, especially in the NSTEACS group was not revascularised. Clinical and/or anatomic reasons such as non-obstructive coronary heart disease, or coronary lesions not amenable to intervention, as well as patient and system delays for STEMI/NC ACS, may explain these revascularisation proportions.

\section{1 | Limitations}

Given the retrospective nature of this study and the data sources, the validity of the conclusions relies on the accuracy and completeness of the original documentation. Although we considered the main confounding variables at the individual and hospital level, no detailed information on socioeconomic status, clinical presentation and time delays was available.

\section{5 | CONCLUSIONS}

In a real-life setting, women with STEMI/NC ACS are less likely than men to be managed invasively. No sex differences in reperfusion and revascularisation were observed in both types of ACS, which represents an important achievement, and help to understand the greater declines in CHD mortality observed among women compared to men in Portugal. Further improvements are still necessary, especially in the management of women with STEMI/NC ACS. To reduce inequalities in management of patients with ACS in Portugal, the sex-gap in analysis and report of results from national registries and other data sources must be improved, and patients admitted to hospitals with lower specialisation levels should be included.

\section{ACKNOWLEDGEMENTS}

The authors gratefully acknowledge the collaboration of the hospitals and local researchers who participated in the Eurhobop study: Centro Hospitalar de Vila Nova de Gaia/Espinho (Vasco Gama Ribeiro, Gustavo Pires de Morais), Centro Hospitalar do Porto (Severo Torres, Mário Santos), Centro Hospitalar Cova da Beira (Miguel Castelo Branco), Centro Hospitalar de São João (Sílvia Marta Oliveira), Hospital de Faro (Ilídio de Jesus, Jorge Mimoso), Hospital Pedro Hispano (Filomena Monteiro), Unidade Hospitalar de Bragança (Domingos Fernandes), Centro Hospitalar do Alto Ave (João Almeida, Filipa Canário Almeida, Francisco Castro Ferreira), Centro Hospitalar de Lisboa Norte (António Nunes Diogo, Maria José Correia), Hospital de Santo André - Leiria (João Morais, Sidarth Pernencar).

\section{DISCLOSURES}

The authors stated that they had no interests which might be perceived as posing a conflict or bias.

\section{FUNDING}

This work was supported by Executive Agency for Health and Consumers (2008 1312 - EURHOBOP) and Unidade de Investigação em Epidemiologia - Instituto de Saúde Pública da Universidade do Porto (EPIUnit) (POCI-01-0145-FEDER-006862; Ref.UID/DTP/04750/2013).

\section{AUTHOR CONTRIBUTIONS}

Carla Araújo participated in data collection, collaborated in the field work, raised the hypotheses, analysed and interpreted the data and drafted the first version of the manuscript. Marta Pereira participated in data collection, collaborated in the field work, analysed and interpreted the data and revised the final version of the manuscript. Olga Laszczyńska analysed and interpreted the data, and revised the final version of the manuscript. Paula Dias interpreted the data and revised the final version of the manuscript. Ana Azevedo coordinated the Portuguese collaboration in EURHOBOP study, raised the hypotheses, interpreted the data and revised the final version of the manuscript. Each author participated sufficiently in the work to take public responsibility for its content and all authors approved the final version of the manuscript.

\section{ORCID}

Carla Araújo iD http://orcid.org/0000-0003-4279-9570

\section{REFERENCES}

1. Pereira M, Azevedo A, Lunet N, et al. Explaining the decline in coronary heart disease mortality in Portugal between 1995 and 2008. Circ Cardiovasc Qual Outcomes. 2013;6:634-642.

2. Hansen KW, Soerensen R, Madsen M, et al. Developments in the invasive diagnostic-therapeutic cascade of women and men with acute coronary syndromes from 2005 to 2011: A nationwide cohort study. BMJ Open. 2015;5:e007785.

3. Moreira D, Marmelo B, Delgado A, et al. A conservative strategy in nonST-segment elevation myocardial infarction - constraints and prognosis: The situation in Portugal. Rev Port Cardiol. 2015;34:315-328.

4. Pereira H, Campante Teles R, Costa M, et al. Trends in primary angioplasty in Portugal from 2002 to 2013 according to the Portuguese National Registry of Interventional Cardiology. Rev Port Cardiol. 2016;35:395-404.

5. Araujo C, Pereira M, Viana M, et al. Regional variation in coronary heart disease mortality trends in Portugal, 1981-2012. Int J Cardiol. 2016;224:279-285.

6. Pereira M, Araujo C, Dias P, et al. Age and sex inequalities in the prescription of evidence-based pharmacological therapy following an acute coronary syndrome in Portugal: The EURHOBOP study. Eur J Prev Cardiol. 2014;21:1401-1408.

7. Barros V, Pereira M, Araujo C, Braga P, Azevedo A. Use of drugeluting versus bare-metal stents after an acute coronary syndrome in Portugal: The EURHOBOP study. Rev Port Cardiol. 2015;34:449-456. 
8. Graham G. Acute coronary syndromes in women: Recent treatment trends and outcomes. Clinical Medicine Insights Cardiology. 2016;10:1-10.

9. Degano IR, Subirana I, Torre M, et al. A European benchmarking system to evaluate in-hospital mortality rates in acute coronary syndrome: The EURHOBOP project. Int J Cardiol. 2015;182:509-516.

10. Andre R, Bongard V, Elosua R, et al. International differences in acute coronary syndrome patients' baseline characteristics, clinical management and outcomes in Western Europe: The EURHOBOP study. Heart. 2014;100:1201-1207.

11. Roffi M, Patrono C, Collet JP, et al. 2015 ESC Guidelines for the management of acute coronary syndromes in patients presenting without persistent ST-segment elevation: Task Force for the Management of Acute Coronary Syndromes in Patients Presenting without Persistent ST-Segment Elevation of the European Society of Cardiology (ESC). Eur Heart J. 2016;37:267-315.

12. Ibanez B, James S, Agewall S, et al. ESC Guidelines for the management of acute myocardial infarction in patients presenting with ST-segment elevation: The Task Force for the management of acute myocardial infarction in patients presenting with ST-segment elevation of the European Society of Cardiology (ESC). Eur Heart J. 2017. doi: 101093/eurheartj/ehx393 [Epub ahead of print].

13. Nutritional anaemias. Report of a WHO scientific group. World Health Organ Tech Rep Ser. 1968;405:5-37.

14. Levey AS, Coresh J, Greene T, et al. Expressing the modification of diet in renal disease study equation for estimating glomerular filtration rate with standardized serum creatinine values. Clin Chem. 2007;53:766-772.

15. Kolte D, Khera S, Aronow WS, et al. Regional variation across the United States in management and outcomes of ST-elevation myocardial infarction: Analysis of the 2003 to 2010 nationwide inpatient sample database. Clin Cardiol. 2014;37:204-212.

16. Menon V, Rumsfeld JS, Roe MT, et al. Regional outcomes after admission for high-risk non-ST-segment elevation acute coronary syndromes. Am J Med. 2006;119:584-590.

17. Magalhaes $P$, Mateus $P$, Carvalho $S$, et al. Relationship between treatment delay and type of reperfusion therapy and mechanical complications of acute myocardial infarction. Eur Heart J Acute Cardiovasc Care. 2016;5:468-474.
18. Pepine CJ, Ferdinand KC, Shaw LJ, et al. Emergence of nonobstructive coronary artery disease: A woman's problem and need for change in definition on angiography. J Am Coll Cardiol. 2015;66:1918-1933.

19. Reynolds HR, Srichai MB, Iqbal SN, et al. Mechanisms of myocardial infarction in women without angiographically obstructive coronary artery disease. Circulation. 2011;124:1414-1425.

20. Heidenreich PA, Shlipak MG, Geppert J, McClellan M. Racial and sex differences in refusal of coronary angiography. Am J Med. 2002;113:200-207.

21. Golden KE, Chang AM, Hollander JE. Sex preferences in cardiovascular testing: The contribution of the patient-physician discussion. Acad Emerg Med. 2013;20:680-688.

22. Levesque JF, Harris MF, Russell G. Patient-centred access to health care: Conceptualising access at the interface of health systems and populations. Int J Equity Health. 2013;12:18.

23. Santos JF, Aguiar C, Gavina C, Azevedo P, Morais J. Portuguese Registry of Acute Coronary Syndromes: Seven years of activity. Rev Port Cardiol. 2009;28:1465-1500.

24. Widimsky P, Wijns W, Fajadet J, et al. Reperfusion therapy for ST elevation acute myocardial infarction in Europe: Description of the current situation in 30 countries. Eur Heart J. 2010;31:943-957.

25. Canto JG, Rogers WJ, Goldberg RJ, et al. Association of age and sex with myocardial infarction symptom presentation and in-hospital mortality. JAMA. 2012;307:813-822.

26. Windecker S, Kolh P, Alfonso F, et al. 2014 ESC/EACTS Guidelines on myocardial revascularization: The Task Force on Myocardial Revascularization of the European Society of Cardiology (ESC) and the European Association for Cardio-Thoracic Surgery (EACTS)Developed with the special contribution of the European Association of Percutaneous Cardiovascular Interventions (EAPCI). Eur Heart J. 2014;35:2541-2619.

How to cite this article: Araújo C, Pereira M, Laszczyńska O, Dias P, Azevedo A. Sex-related inequalities in management of patients with acute coronary syndrome-results from the EURHOBOP study. Int J Clin Pract. 2018;72:e13049. https://doi. org/10.1111/ijcp.13049 